\title{
HR diagram and asteroseismic analysis of models for $\beta$ Hydri
}

\author{
J. Fernandes ${ }^{1}$ and M. J. P. F. G. Monteiro ${ }^{2,3}$ \\ ${ }^{1}$ Observatório Astronómico da Universidade de Coimbra e Departamento de Matemática, FCTUC, Portugal \\ e-mail: jmfernan@mat.uc.pt \\ 2 Departamento de Matemática Aplicada, Faculdade de Ciências da Universidade do Porto, Portugal \\ ${ }^{3}$ Centro de Astrofísica da Universidade do Porto, Rua das Estrelas, 4150-762 Porto, Portugal
}

Received 29 April 2002 / Accepted 14 November 2002

\begin{abstract}
A detailed review of the models for the star $\beta$ Hydri is done by adjusting the evolutionary sequences to the most recent bolometric, spectroscopic and astrometric data. The dependence of the solution on some of the relevant modelling parameters is analysed and the degeneracy of the solution using the HR diagram analysis discussed.

Recent ground-based observations, and the forthcoming asteroseismic missions, can provide detailed oscillation spectra for this star. The seismic data can be used as a complementary constraint on the models. We analyse the seismic properties of the star and discuss the viability of using such complementary tests in order to clarify some of the degeneracy of the model solutions. Preliminary results on the fundamental parameters of $\beta$ Hydri are presented and the expected seismic behaviour of the star is described.
\end{abstract}

Key words. stars: evolution - stars: fundamental parameters - stars: individual: $\beta$ Hydri - stars: oscillations

\section{Introduction}

$\beta$ Hydri, $\beta$ Hyd (HD 2151, HR 98, HIP 2021), is a G2 IV star in the neighbourhood of the Sun belonging to the old disc of the Galaxy. This evolved star is frequently seen as the distant future of the Sun (Dravins et al. 1993). So $\beta$ Hyd has been extensively studied in different aspects placing it in a very interesting place among the solar type stars: the tentative detection of extra-solar planets (Skuljan et al. 2000), the abnormal high lithium abundance for an evolved star - 10 times the solar value (cf. Randich et al. 1999), atmospheric and chromospheric activity (Chmielewski 2000), etc. On the other hand, thanks to HIPPARCOS data and to recent determinations of the observed bolometric flux, as well as spectroscopic evaluations of the atmospheric parameters, the position of this star in the Hertzsprung-Russel diagram (HRD) is known to better than $3 \%$. It can, therefore, be used to test the stellar evolution theory and constraints on the physical description of the stellar interiors. Using modern stellar evolutionary models, Dravins et al. (1998) concluded that the mass of the star should be around $1.05-1.22$ of the solar mass, with an age of 6.7 Gyr. This value is lower than the previous determination of 8 to $10 \mathrm{Gyr}$, thanks to the new distance of $7.5 \mathrm{pc}$ measured by HIPPARCOS compared to the old $6.7 \mathrm{pc}$ obtained from the ground (van Altena et al. 1995).

However, Dravins et al. (1998) discuss the modelling in the HRD of $\beta H y d$ based on the lower value of the metallicity of

Send offprint requests to: M. J. Monteiro,

e-mail: mjm@astro.up.pt
$[\mathrm{Fe} / \mathrm{H}]=-0.2$. Further observations indicated that this value should be slightly higher, near -0.1 . On the other hand it can be expected that stars with mass higher than the solar value develop a convective core during some part of their evolution (cf. Guenther \& Demarque 2000), so the effect of the overshooting must be checked.

On the other hand it is well known that the analysis of the HRD position of a single star, other than the Sun, is inconclusive because the number of free parameters is higher that the observables. Models with different sets of free parameters can easily satisfy the observations.

Recently, Bedding et al. (2001) and Carrier et al. (2001) confirmed the presence of solar type oscillations in $\beta \mathrm{Hyd}$. A clear excess power has been measured independently for this star at about $1000 \mu \mathrm{Hz}$. An extensive seismic analysis can only be done if a significant number of individual mode frequencies is measured, which has not been done yet. However, information associated with the frequency separation, as detected in this case, is already a useful observational test that can complement the classical HRD analysis (eg. Morel et al. 2000 and references therein).

In this work we will revise the present models of $\beta H y d$ by using the latest updates of the physics and adjusting the most recent spectroscopic, photometric and astrometric data for this star. We further aim at studying how seismic data for this star can be used to solve some of the degeneracy associated with the model parameters used to adjust the observed luminosity and effective temperature. In order to do so we address the problem by considering different sets of seismic data. We start by 
simply using the large frequency separation, going up to the best case of having a significant set of individual mode frequencies which can be identified and whose frequency is measured with high accuracy.

With such an analysis we attempt to provide state of the art models for the star and how the seismic data, which we expect to have in the near future, can help to resolve some of the uncertainties that cannot be addressed with the "classical" observational data.

\section{Observations}

A summary of the presently available data on $\beta H y d$ is described here. We have chosen to divide the observations by "classical" (spectrometric, bolometric and astrometric) and seismic data, in order to reflect the structure of the work presented in the following sections.

\subsection{Bolometric, spectrometric and astrometric data}

The luminosity of a star can be determined through the knowledge of the observed bolometric flux and the distance, so avoiding the bolometric correction. Recently Blackwell et al. (1998) and DiBenedetto (1998) found the same value for the bolometric flux for $\beta$ Hyd: $(2.02 \pm 0.05) \times 10^{-9} \mathrm{~W} / \mathrm{m}^{2}$, using the InfraRed Flux Method (IRFM). The HIPPARCOS mission measured the parallax as being $133.78 \pm 0.51$ mas (Perryman et al. 1997). By combining these values, a luminosity for $\beta \mathrm{Hyd}$ of $3.53 \pm 0.10$ times the solar luminosity $\left(L_{\odot}\right)$ is found.

Many authors have claimed that the effective temperature of $\beta H y d$ is identical to the solar value (see Dravins et al. 1993 for details). Recently DiBenedetto (1998), using the IRFM method, found $5774 \pm 60 \mathrm{~K}$, confirming the solar value of the temperature for this star. We adopt this value, consistent with the above bolometric flux and in excellent agreement with Favata et al. (1997), who found $5775 \mathrm{~K}$ using the $(R-I)$ colour index.

In the last version of the Catalogue of $[\mathrm{Fe} / \mathrm{H}]$ determinations, the edition by Cayrel de Strobel et al. (2001), we can find reference to eight metallicity values for $\beta \mathrm{Hyd}$. This catalogue only includes determinations that have been submitted to detailed spectroscopic analysis from the ground. The two most recent determinations using high resolution spectra give identical values: $-0.12 \pm 0.04$ (Favata et al. 1997) and $-0.11 \pm 0.07$ (Castro et al. 1999). We adopt the average of these determinations (with a conservative error bar), $[\mathrm{Fe} / \mathrm{H}]=-0.12 \pm 0.07$, a value slightly higher than the -0.2 assumed previously (Dravins et al. 1998). For Pop. I stars the abundance of metals $Z$ is related to the $\mathrm{Fe} / \mathrm{H}$ by $[\mathrm{Fe} / \mathrm{H}]=\log (Z / X)-\log (Z / X)_{\odot}$, where $(Z / X)_{\odot}=0.0245$ is the ratio of the solar mixture (Grevesse \& Noels 1993). This gives $(Z / X)=0.019 \pm 0.003$ for $\beta H y d$.

The rotation has also an influence on the HRD position (e.g. Maeder \& Meynet 2000). Dravins and Nordlund (1990), by fitting line profiles from hydrodynamic models of the atmosphere, found $(v \sin i)=2 \pm 1 \mathrm{~km} \mathrm{~s}^{-1}$. Thus, we can expect this star to be a slow rotator - a non-rotating and spherical stellar model seems to be appropriate.
Table 1. Observational data for $\beta H y d$ used in this work. For the details see the text.

\begin{tabular}{|c|c|c|}
\hline Observable & Value & Source \\
\hline$L / L_{\odot}$ & $3.53 \pm 0.10$ & $\begin{array}{l}\text { Blackwell et al. (1998) } \\
\text { DiBenedetto (1998) }\end{array}$ \\
\hline$T_{\text {eff }}(\mathrm{K})$ & $5774 \pm 60$ & DiBenedetto (1998) \\
\hline$Z / X$ & $0.019 \pm 0.003$ & $\begin{array}{l}\text { Favata et al. (1997) } \\
\text { Castro et al. (1999) }\end{array}$ \\
\hline$\Delta v(\mu \mathrm{Hz})$ & $56.2 \pm 2.0$ & Bedding et al. (2001) \\
\hline
\end{tabular}

\subsection{Seismic data}

Seismic data for this star has been found by Bedding et al. (2001) and Carrier et al. (2001) indicating a clear excess power around $700 \mu \mathrm{Hz}<v_{0}<1000 \mu \mathrm{Hz}$, corresponding to a signal of about $0.40 \mathrm{~m} \mathrm{~s}^{-1}$ peak amplitude. Bedding et al. (2001) cite the value of $56.2 \mu \mathrm{Hz}$ as the most likely value of the large frequency separation. Carrier et al. (2001) found a value of $58 \mu \mathrm{Hz}$ (with an uncertainty of about $2 \mu \mathrm{Hz}$ ) which is compatible with the previous result.

In order to illustrate how seismic data can be used to provide stringent constraints on the models we will also consider here the possibility of having accurate seismic data. Such data will be available from scheduled space missions like MONS (Kjeldsen et al. 2000), MOST (Matthews et al. 2000) and COROT (Baglin et al. 1998), or missions like Eddington (Favata et al. 2002). From these projects we expect to have a wide set (more than 20) of individual mode frequencies for $l=0,1,2$ (and eventually $l=3$ ), with an accuracy of $\sigma(v) \leq 0.1 \mu \mathrm{Hz}$. Here we address what such data will provide in terms of complementing the already available classical data for this star.

\section{Input physics in the theoretical stellar models}

The stellar evolution calculations were computed with the CESAM code version 3. (Morel 1997), running at the Coimbra Observatory.

Details on the physics of these models can be found in Lebreton et al. (1999). Here we summarise: the CEFF equation of state, including Coulomb corrections to the pressure (Eggleton et al. 1973; Christensen-Dalsgaard 1991); nuclear reactions rates given by Caughlan \& Fowler (1988); solar mixture from Grevesse \& Noels (1993); OPAL opacities (Iglesias \& Rogers 1996) complemented at low temperatures by opacities data from Alexander \& Ferguson (1993) following a prescription of Houdek \& Rogl (1996); the atmosphere is described with an Eddington $T(\tau)$-law; convection is treated according to the mixing-length theory from Bohm-Vitense (1958) leaving the mixing-length $\left(\alpha \times H_{\mathrm{p}}\right)$ and the amount of the core overshooting $\left(\alpha_{\mathrm{ov}} \times H_{\mathrm{p}}\right)$ as unknowns; $\alpha$ and $\alpha_{\mathrm{ov}}$ are parameters where $H_{\mathrm{p}}$ is the local pressure scale height.

With this input physics the solar model fits the observed luminosity and radius, in one part in 10000 , with $\alpha=1.63$, helium abundance $Y=0.268$ and $Z=0.0175$ for the common 
Table 2. Evolutionary sequences of models. $M$ is the mass of the model (in units of the solar mass $M_{\odot}$ ), $Y$ is the initial helium abundance, $Z=0.014, \alpha$ the mixing length parameter and $\alpha_{\mathrm{ov}}$ is the parameter associated with the extension of the overshooting region at the top of the convective core.

\begin{tabular}{lcccc}
\hline \hline Model & $M / M_{\odot}$ & $Y$ & $\alpha$ & $\alpha_{\mathrm{ov}}$ \\
\hline$S_{0}$ & 1.10 & 0.27 & 1.4 & 0.25 \\
$S_{a 1}$ & 1.00 & - & - & - \\
$S_{a 2}$ & 1.20 & - & - & - \\
$S_{b 1}$ & - & 0.24 & - & - \\
$S_{b 2}$ & - & 0.30 & - & - \\
$S_{c 1}$ & - & - & 1.6 & - \\
$S_{c 2}$ & - & - & 1.8 & - \\
$S_{d 1}$ & - & - & - & 0.00 \\
$S_{d 2}$ & - & - & - & 0.15 \\
\hline
\end{tabular}

accepted solar age of 4.6 Gyr (Dziembowski et al. 1999) and the ratio of the solar mixture from Grevesse \& Noels (1993).

\section{HR diagram analysis}

\subsection{Standard evolutionary models}

It is well known that the knowledge of the HRD position $L$ and $T_{\text {eff }}-$ is not sufficient to define all stellar parameters. In fact, any point in the HRD depends on stellar mass, helium, metallicity, age and some free parameters on the input physics of stellar models. This problem can be solved for the Sun, whose mass, metallicity and age are known (e.g. Christensen-Dalsgaard 1982) and for some binary systems where individual masses are available (e.g. Noels et al. 1991; Fernandes et al. 1998). For a single star, other than the Sun, commonly there are more unknowns than observables. For $\beta H y d$ we take the metallicity as an observable - we then have three observables $\left(Z / X, L, T_{\text {eff }}\right)-$ for six unknowns $(Y, Z, M$, $\left.t_{\star}, \alpha, \alpha_{\mathrm{ov}}\right)$.

We construct our reference model by tuning the parameters in order to reproduce the observations on the HRD taking into account some constraints: mass - different authors have claimed that the mass of $\beta \mathrm{Hyd}$ should be around 1.0-1.2 $\mathrm{M}_{\odot}$ (Dravins et al. 1998, and references therein); age - for a long time that it has been known that $\beta$ Hyd is an evolved star on the sub-giant stage, so typically the age should be higher than the solar one; overshooting - stellar models of stars with mass higher than the solar value show the presence of a convective core, at least at the beginning of their evolution. This seems to be confirmed by observations (Ribas et al. 2000).

Our reference model, hereafter $S_{0}$, has the following characteristics: $M=1.1 M_{\odot}, Y=0.27, Z=0.014, \alpha=1.4$ and $\alpha_{\mathrm{ov}}=0.25$. This model reproduces the observed quantities $\left(L, T_{\text {eff }}\right)$ as well as $1 \%$ for an age of $6.82 \mathrm{Gyr}$.

We have also studied the sensitivity of model $S_{0}$ to the variation of the parameters. In Table 2 we list the computed models for different parameters (when it is not indicated, the model characteristics are the same as for $S_{0}$ ). In Figs. 1 and 3 we plot

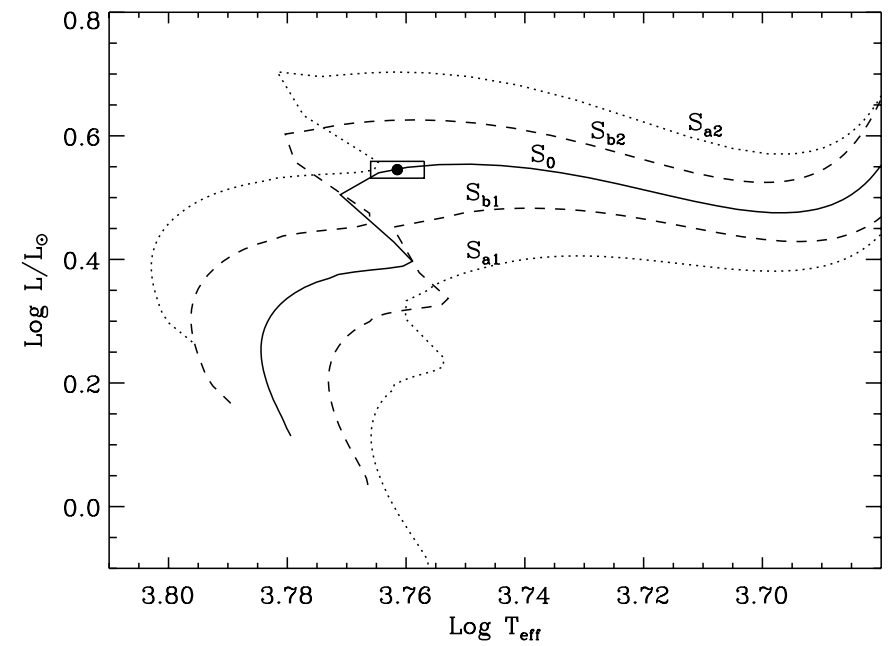

Fig. 1. Evolutionary tracks for the test models; $S_{0}$ (full line), $S_{a 1}$ and $S_{a 2}$ (dotted lines), $S_{b 1}$ and $S_{b 2}$ (dashed lines), listed in Table 2, are shown in order to illustrate how sensitive the evolution is to stellar mass and helium.

the corresponding evolutionary tracks when each parameter is changed, relative to model $S_{0}$, and the observational error box (see Sect. 2).

It is clear from Fig. 1 that the HRD position of the models is strongly dependent on mass and helium. As it is well known, both helium and mass have similar effects on the mainsequence and sub-giant phases: the increase of helium or mass produces an increase in luminosity and effective temperature.

However if we concentrate on the sub-giant phase, for a fixed $T_{\text {eff }}$ and leaving the age free, we can infer that an increase in helium can be compensated by a decrease of the mass in order to get the same position in the HRD (e.g. Lebreton et al. 1993): a helium-mass degeneracy. This is so because the evolution in this regime is almost parallel to the $T_{\text {eff }}$-axis, as expected for an evolution characterised almost solely by the size of the central helium core. So, we can find the pairs $(M, Y)$ that, for a fixed value of $T_{\text {eff }}$ (e.g. the observed value), reproduce the observed luminosity, $L_{\mathrm{obs}}$, using a solar calibration-like formula (Christensen-Dalsgaard 1982) for $Z=0.014$ :

$$
\begin{aligned}
\log L_{\mathrm{obs}}= & \log L_{\mathrm{ref}}+\left(\frac{\Delta \log L}{\Delta Y}\right)_{M} \times\left(Y-Y_{\mathrm{ref}}\right) \\
& +\left(\frac{\Delta \log L}{\Delta M}\right)_{Y} \times\left(M-M_{\mathrm{ref}}\right) .
\end{aligned}
$$

The subscript "ref" indicates a stellar evolutionary model that falls inside the error bar in luminosity, e.g. our reference model $S_{0}$. Assuming the linearity of the variation of $\log L$ with helium and mass, we found

$\left(\frac{\Delta \log L}{\Delta Y}\right)_{M} \approx 2.72$ and $\left(\frac{\Delta \log L}{\Delta M}\right)_{Y} \approx \frac{1.82}{M_{\odot}}$

Finally, Eq. (1) becomes

$Y( \pm 0.01)=1.00-0.67 \times \frac{M}{M_{\odot}}$

Equation (3) reproduces the observed luminosity of $\beta \mathrm{Hyd}$ to within $1 \%$ for the range of mass between $1.0 M_{\odot}$ and $1.15 M_{\odot}$, 


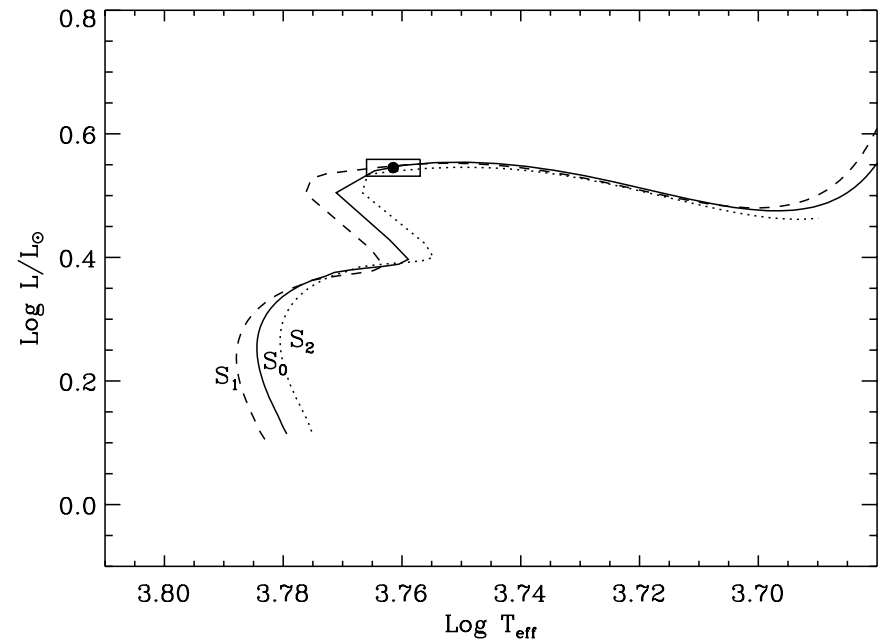

Fig. 2. Mass-luminosity degeneracy - evolutionary tracks for the test models $S_{0}$ (full line), $S_{1}$ (dashed line) and $S_{2}$ (dotted line) - see Table 3.

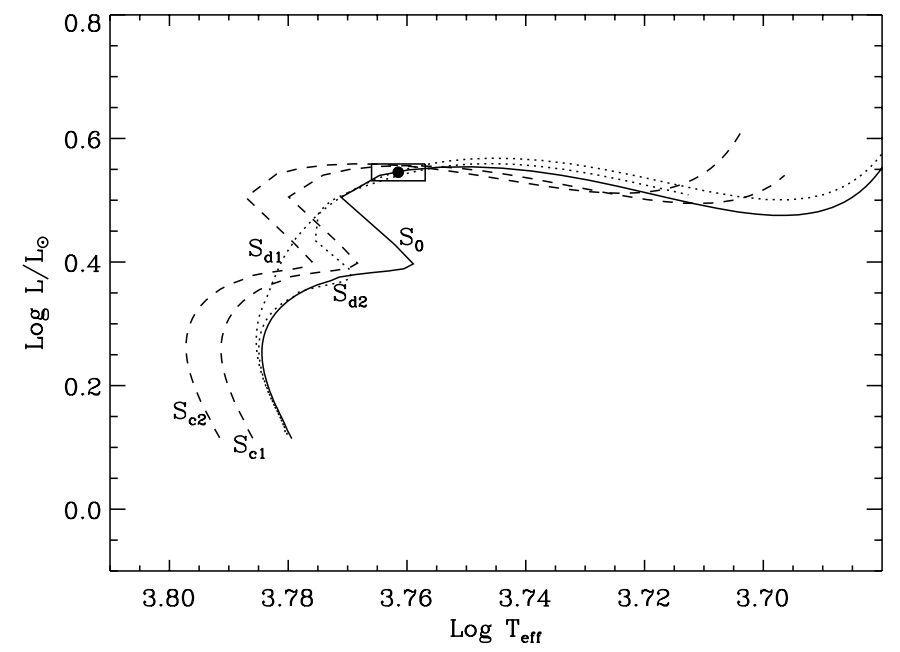

Fig. 3. Evolutionary tracks for the test models $S_{c 1}$ and $S_{c 2}$ (different values of $\alpha$-dashed lines), $S_{d 1}$ and $S_{d 2}$ (different values of $\alpha_{\text {ov }}$ - dotted lines), compared with $S_{0}$. These evolutionary sequences illustrate how sensitive is the evolution to the convection parameters $\alpha$ and $\alpha_{\mathrm{ov}}$.

and the corresponding $Z / X$ falls in the error bar of the observed value (Fig. 2).

Concerning the sensitivity of the models to the convection parameters $\left(\alpha, \alpha_{\mathrm{ov}}\right)$, Fig. 3 shows a curious situation: the error box of $\beta H y d$ is precisely in the region where all evolutionary tracks intercept each other. No distinction is possible. So by the simple analysis of the HRD, it is not possible to decide about (i) the value of the mixing length parameter and (ii) the value of the overshooting. Eventual improvements on the error box of ( $\left.L, T_{\text {eff }}\right)$ will not change the situation because the changes in convection parameters produce mainly a horizontal variation in the HRD.

Similar conclusions can be obtained regarding mass and helium, as a result of the degeneracy between mass and helium that we have discussed above.

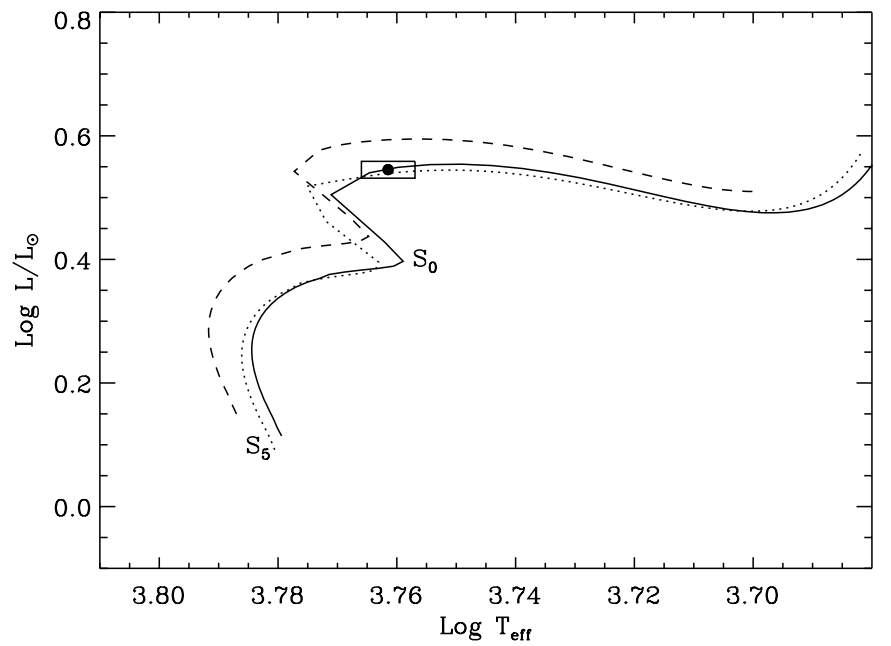

Fig. 4. Evolutionary track for a test model calculated with the same parameters as $S_{0}$, except for $Z / X=0.016$ (dashed line). In order to compare, both the reference model $S_{0}$, and the evolutionary sequence for model $S_{5}$ (with mass $1.07 M_{\odot}-$ see text) are shown.

\subsection{Changing the observed $Z / X$}

As indicated above, old metallicity determinations give a value lower than the one used in the calculation of $S_{0}$, so we tested our models for a metallicity equal to the lower extreme of the error bar; $[\mathrm{Fe} / \mathrm{H}]=-0.19$ or $Z / X=0.016$. Using the same $S_{0}$ helium value it follows that $Z=0.012$. We compute a model with the same characteristics of $S_{0}$ but using a different $Z$. Figure 4 shows the effect of a change in metal abundance. The variation in the HRD is similar to an increase in helium or mass. A lower value of about $0.03 M_{\odot}$ in mass (or 0.02 in helium) is sufficient to return the evolutionary track to agreement with the observations (model $S_{5}$, see Table 3 ).

\subsection{What about diffusion?}

The solar standard model can be put in closer agreement with Helioseismology if the efficiency of the microscopic diffusion of helium and metals is considered (e.g. Bahcall \& Pinsonneault 1995). The classical formalism for the treatment of the gravitational settling of chemical elements seems also to put in agreement observations and theory for nearby low mass stars of Population II (Lebreton et al. 1999) and for old globular clusters (e.g. Castellani et al. 1997). On the other hand the present formalism fails to explain the depletion of light elements in the surface of the Sun and stars, such as the lithium (e.g. Morel et al. 2000), as well as to explain the photospheric abundances in stars more massive than the Sun (Richer et al. 2000). Other descriptions are strongly needed; for example the turbulent mixing just under the convection zone (Montalban \& Schatzman 2000).

Because $\beta H y d$ has a mass close to the solar value and it is a evolved star, we choose to test the effect of diffusion using the same formalism that has been tested and well established for the Sun (Morel et al. 1997).

We start by computing a model with the exact characteristics of $S_{0}$ but including the diffusion of helium and metals 
Table 3. Models of $\beta H y d$ having the observed luminosity and effective temperature (within the error bars), but using different combinations of the modelling parameters in order to adjust the model to the observations. Model $S_{6}$ also includes diffusion of helium and metals. The quantity $Z / X$ corresponds to the value at the surface of the model.

\begin{tabular}{ccccccccccc}
\hline \hline Mod & $M / M_{\odot}$ & $Y$ & $\alpha$ & $\alpha_{\text {ov }}$ & $Z / X$ & $t(\mathrm{Gyr})$ & $R / R_{\odot}$ & $L / L_{\odot}$ & $T_{\text {eff }}(K)$ & \\
\hline$S_{0}$ & 1.10 & 0.27 & 1.4 & 0.25 & $0.019(6)$ & 6.820 & 1.899 & 3.540 & 5751 & \\
$S_{1}$ & 1.05 & 0.30 & 1.4 & 0.25 & $0.020(4)$ & 6.414 & 1.878 & 3.529 & 5778 & \\
$S_{2}$ & 1.15 & 0.23 & 1.4 & 0.25 & $0.018(5)$ & 7.125 & 1.883 & 3.477 & 5749 & \\
$S_{3}$ & 1.10 & 0.27 & 1.4 & 0.00 & $0.019(6)$ & 6.653 & 1.878 & 3.520 & 5775 & \\
$S_{4}$ & 1.10 & 0.27 & 1.8 & 0.25 & $0.019(6)$ & 7.038 & 1.908 & 3.595 & 5760 & \\
$S_{5}$ & 1.07 & 0.27 & 1.4 & 0.25 & $0.016(7)$ & 6.926 & 1.830 & 3.443 & 5818 & \\
$S_{6}$ & 1.10 & 0.28 & 1.4 & 0.25 & $0.022(7)$ & 0.000 & 1.036 & 1.239 & 5984 & $Z$ ZAMS \\
$S_{6}$ & 1.10 & 0.23 & 1.4 & 0.25 & $0.018(8)$ & 6.881 & 1.878 & 3.477 & 5757 & \\
\hline
\end{tabular}

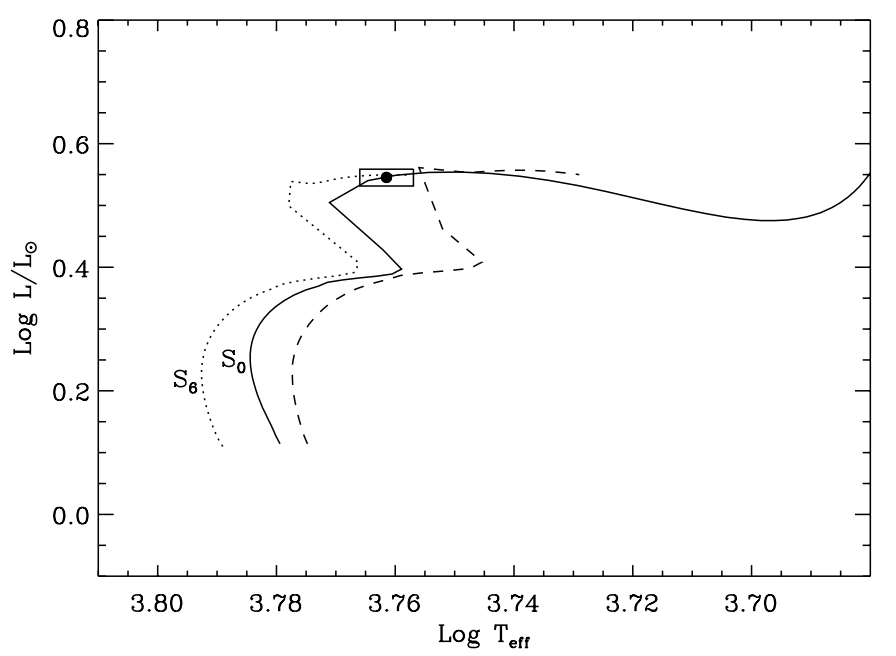

Fig. 5. Evolutionary track for a test model calculated including the diffusion of chemical elements (dashed line). The reference model $S_{0}$ is also shown (full line). The model $S_{6}$ (dotted line) also includes diffusion, and reproduces the observed $\left(Z / X, T_{\text {eff }}, L\right)$ for an age of $6.88 \mathrm{Gyr}$.

(Fig. 5). As expected, due to the increase of the hydrogen opacity in the external layers of the star, the evolutionary track with diffusion becomes cooler than $S_{0}$. Thanks to the depletion of the helium and metals, when the evolutionary track crosses the error box, around an age of $6.9 \mathrm{Gyr}$, the $Z / X$ at the surface is no longer the observed value 0.019 , but instead 0.016 . This can still be considered consistent with the observations as this value is the lower extreme of the $Z / X$ error bar. However, in order to try to find the best model, we recomputed a new evolutionary track that reproduces the observed $Z / X$, as well as $\left(L, T_{\text {eff }}\right)-$ this is model $S_{6}$.

\section{Asteroseismic analysis}

By considering different sets of seismic data that are (or may become) available for this star we discuss here the constraints that we may impose on the models for this star. With such set of tests we aim at defining what aspects of the physics discussed above can be separated, lifting some of the degeneracy we have found with the HRD analysis.
Table 4. Models of different ages corresponding to the evolutionary sequence producing the reference model $S_{0}$.

\begin{tabular}{lcccc}
\hline \hline Model & $R / R_{\odot}$ & $L / L_{\odot}$ & $t(\mathrm{Gyr})$ & $X_{\mathrm{c}}$ \\
\hline$S_{x 7}$ & 1.051 & 1.301 & 0200.0 & 0.704 \\
$S_{x 5}$ & 1.200 & 1.776 & 3000.0 & 0.490 \\
$S_{x 2}$ & 1.477 & 2.378 & 5759.1 & 0.174 \\
$S_{0}$ & 1.899 & 3.540 & 6819.6 & 0.000 \\
\hline
\end{tabular}

Table 3 lists the properties of the models we consider here. They all have the observed value of the luminosity and effective temperature (within the error bars), but use different combinations of the stellar mass and initial helium abundance, and other modelling parameters.

We will now apply some of the seismic tests available and consider the analysis for different sets of seismic data that have been or will be measured for this star.

\subsection{Seismic properties of $\beta$ Hyd}

$\beta$ Hyd is an evolved star of a mass slightly higher than the solar value. By calculating the linear adiabatic oscillations with degree $l=0,1,2$ for our reference model $S_{0}$ we find that modes with mode order $8<n<24$ have frequencies between $500 \mu \mathrm{Hz}<v_{l n}<1400 \mu \mathrm{Hz}$. Because the expected age for this star places it after the end of the main-sequence evolution the model exhibits the characteristic avoided crossings (Aizenman et al. 1977) in its p-mode spectra. Such behaviour corresponds to modes changing nature as a consequence of the evolution in this advanced evolutionary stage of the core. This behaviour is expected for these stars (see for example Christensen-Dalsgaard et al. 1995 and Morel et al. 2001), making the analysis of their seismic spectra much more difficult.

Radial modes are not expected to be affected, but the frequencies found for $l=1$ and $l=2$ show this characteristic behaviour. It is illustrated in Fig. 6 where the large frequency separation is shown for models of different ages corresponding to the evolutionary sequence that produces our reference model $S_{0}$. Only at the end of the main-sequence, as the central helium core is formed, does the effect of avoided crossings for 


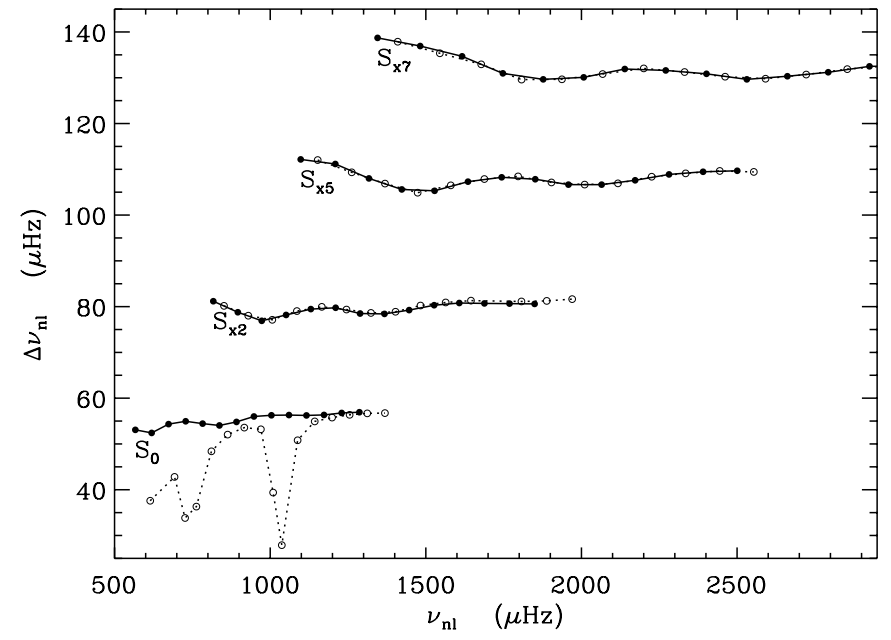

Fig. 6. Large frequency separations (Eq. (4)) for models with the same properties as our reference model but with different ages (from the ZAMS up to the age of model $S_{0}$ ). The open circles are the values of the separation for modes of degree $l=1$, while the closed circles are for $l=0$.

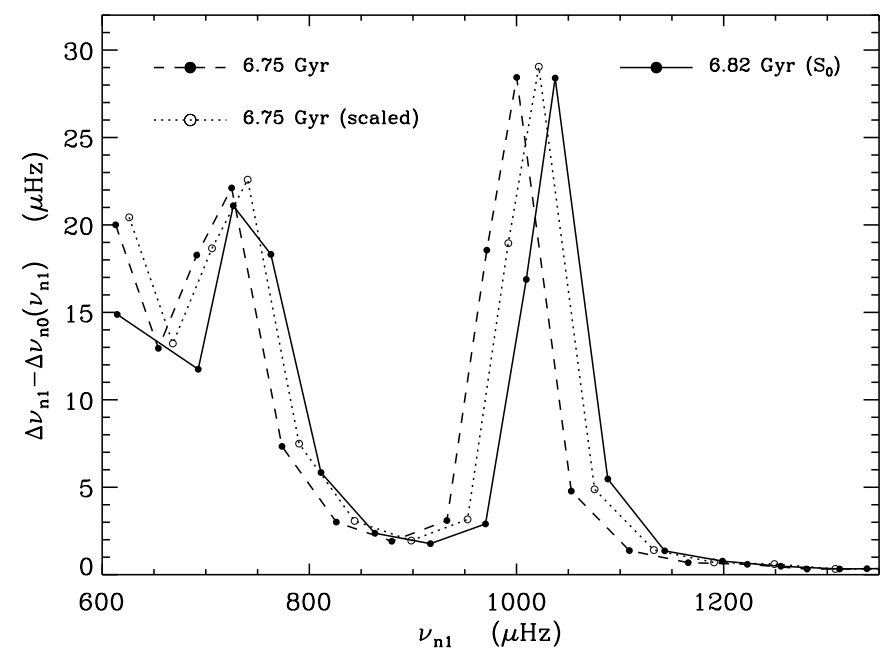

Fig. 7. Differences between the large frequency separations for modes with $l=1$ and modes with $l=0$. The differences are shown for models of different ages: $S_{0}$ (full line) and a younger model (dashed line), as indicated for each curve by the age. Also shown are the differences calculated after scaling the frequencies by a factor as given in Eq. (5) (dotted line) - it illustrates the contribution to the diferences due to the change of the radius of the star with age.

modes of $l=1$ (and $8 \leq n \leq 22$ ) show up as a strong deviation of the separations from its expected asymptotic behaviour.

Figure 7 illustrates how the large frequency separations for modes of degree $\ell=1$ change with time as the mass of the helium core increases. A change in age of about 0.07 Gyr moves the strongest deviation of the separation from the asymptotic behaviour by about $50 \mu \mathrm{Hz}$. Part of this shift $(\sim 55 \%)$ is due to the change of the radius of the star with age. The remaining component results from the change of the relative mass of the helium-core with age.

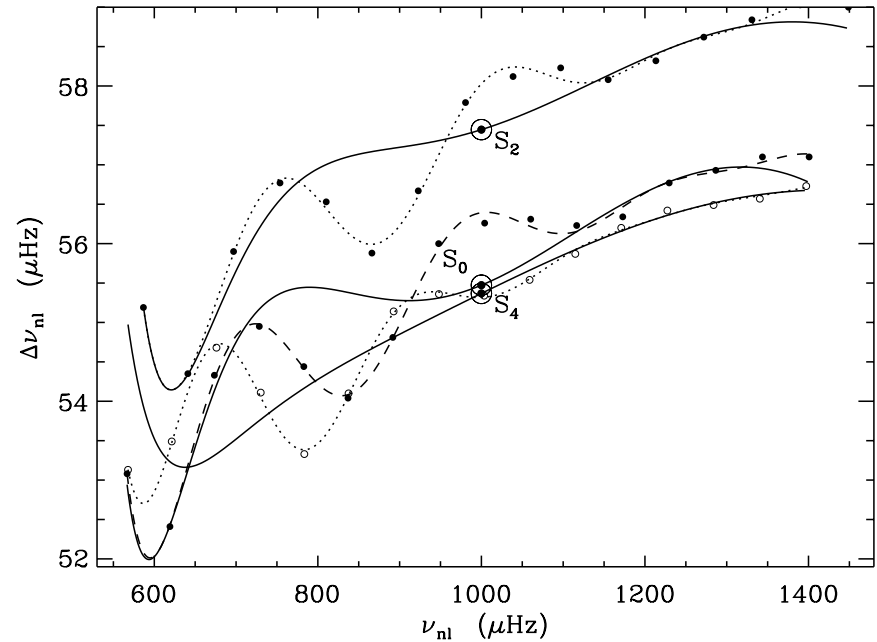

Fig. 8. Large frequency separations (Eq. (4)) for three of the models listed in Table 3 . Only modes with $\ell=0$ are used. The lines correspond to the smooth component which has been used to estimate the value of $\Delta \bar{v}$ (large circles).

\subsection{Seismic tests of the models}

The seismic information that can more easily be identified in the power spectrum of the oscillations is the large separation. It is a regular spacing between frequencies of consecutive mode order $n$ and the same mode degree $l$. Asymptotically this value is expected to scale with $\left(M / R^{3}\right)^{1 / 2}$, where $M$ is the mass of the star and $R$ its radius. Here we calculate the separation (Monteiro et al. 2002) as being its value at a fixed frequency after removing the periodic component present due to the helium ionization zone (Monteiro \& Thompson 1998). Figure 8 illustrates how the separations have been calculated using the modes with degree $l=0$ from the values of

$\Delta v_{n l} \equiv v_{n+1, l}-v_{n, l}$.

The reference value we adopt here to compare the models is defined as being $\Delta \bar{v} \equiv \Delta v_{n l}\left(v_{0}\right)$, where $v_{0}=1000 \mu \mathrm{Hz}$.

The values found for the large frequency separation of the models considered in Table 3 are given in Table 5. There is a clear difference between models that can be as large as $2 \mu \mathrm{Hz}$. Part of that difference is a simple homologous scaling for frequency, resulting from the differences in mass $M$ and radius $R$. Considering that from the observations we can only estimate the radius, the best way to compare the models with the observed seismic data is by considering a "reduced" separation;

$\Delta \bar{v}_{r} \equiv \Delta \bar{v}\left(\frac{R}{R_{0}}\right)^{3 / 2}=\Delta \bar{v}\left(\frac{L}{L_{0}}\right)^{3 / 4}\left(\frac{T_{\mathrm{eff}, 0}}{T_{\mathrm{eff}}}\right)^{3}$

where the subscript " 0 " indicates the values for a reference model (in here, this is model $S_{0}$ ). For a pure homologous scaling of the separation; $\Delta \bar{v}_{r}$ would be a function of stellar mass alone. If we had the correct value for the mass, we should find that $\Delta \bar{v}_{r}=\Delta \bar{v}_{0}$. It is not however a pure homologous scaling as the scaling factor will depend on the physics of the models, namely, the chemical composition and convection, among other factors (known or unknown). 
Table 5. Large frequency separation $\Delta \bar{v}(\mu \mathrm{Hz})$ estimated using the frequencies of modes with degree $l=0$. The quantity $f$ is defined in Eq. (5). The observed value (Bedding et al. 2001) is also show together with the reduced value determined using the data in Table 1.

\begin{tabular}{lcc}
\hline \hline Model & $\Delta \bar{v}$ & $\Delta \bar{v}_{r}$ \\
\hline$S_{0}$ & 55.47 & - \\
$S_{1}$ & 55.17 & 54.27 \\
$S_{2}$ & 57.45 & 56.74 \\
$S_{3}$ & 56.44 & 55.50 \\
$S_{4}$ & 55.37 & 55.75 \\
$S_{5}$ & 57.82 & 54.69 \\
$S_{6}$ & 56.40 & 55.47 \\
\hline$\beta$ Hyd & 56.2 & 55.2 \\
\hline
\end{tabular}

The method developed by Monteiro \& Thompson (1998) can, in principle, be used to resolve the stellar mass-helium abundance degeneracy of the HRD. Such a method isolates from the frequencies a periodic component produced at the second helium ionization zone. The characteristics of the signature are a direct function of the helium abundance near the surface and therefore can be used to provide a complementary constraint when both the mass and the helium abundance are not accurately known. Such a method can be used for lowdegree modes of main-sequence stars. However, for evolved stars, as is the case of $\beta H y d$, we can only take radial modes (see for example Fig. 8) which places strong limitations on the precision with which we may determine the value of $Y$.

We also note that the behaviour of the $\ell=1$ modes, as represented in Figs. 6 and 7, for the case of an evolved star is strongly dependent on age. Therefore it may be used to constrain the age, providing a complementary test on what a fitting parameter is in the HRD analysis. Such a dependence is a direct consequence of the direct dependence of the evolution of the star in this post-main sequence phase on the size of its helium core, when the star evolves almost at constant effective temperatures.

Figure 9 shows the reduced large frequency separation for all models that adjust $\beta$ Hyd in the HRD. The differences between $S_{0}, S_{3}, S_{4}$ and $S_{6}$ are a measure of the deviation from the scaling law used to define $\Delta \bar{v}_{r}$ (Eq. (5)). The change in the mixing length parameter is the strongest. The homology scaling is not directly dependent on the initial helium abundance $Y$ (see model $S_{6}$ ) making the large frequency separation an important indicator of stellar mass, when the helium abundance is not known.

The fact that the reduced large frequency separation is mainly dependent on the mass of the star can be used to infer this quantity from the seismic observations. As an example we consider the local dependence of $\Delta \bar{v}_{r}$ on $M / M_{\odot}$, estimated from the properties of all models listed in Table 3 . In order to do so, and taking into account the relation given in Eq. (4), we consider that

$\frac{M}{M_{\odot}} \simeq \frac{M_{0}}{M_{\odot}}\left(\frac{\Delta \bar{v}_{r}}{D_{v, M}}\right)^{2}$,

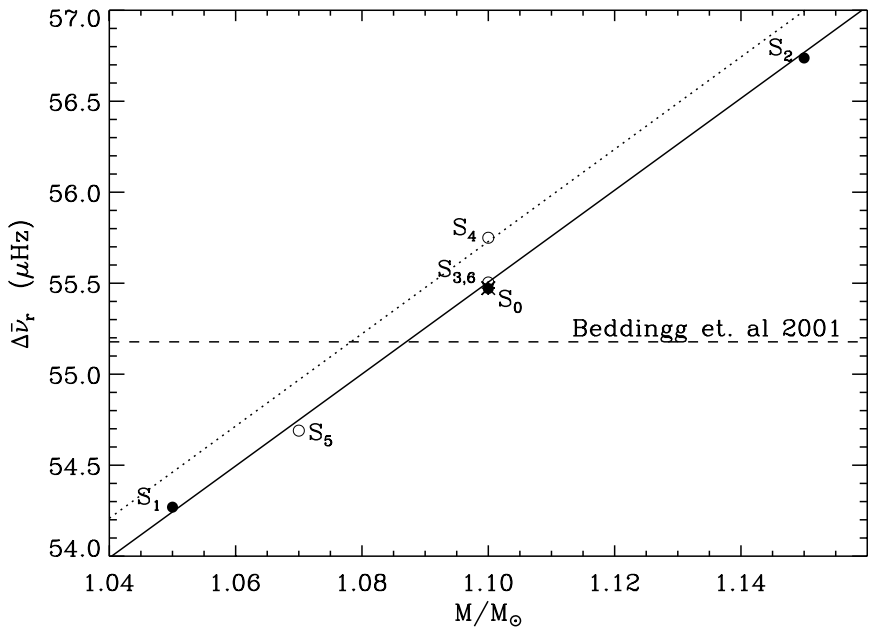

Fig. 9. Reduced large frequency separations (Eq. (5)) for all models listed in Table 3. The continuous line is a least squares fit to all points (see Eq. (6)) while the dotted line illustrates the change introduced in the scaling law by changes in the treatment of convection. The dashed line corresponds to the observational value found by Bedding et al. (2001).

in order to find, by a least squares fitting to the reduced large frequency difference of all models, a value of $D_{v, M} \simeq$ $55.53 \mu \mathrm{Hz}$. This corresponds to the continuous line in Fig. 9. If such an expression is used to estimate the mass from the observed large frequency separation we find that $M / M_{\odot} \simeq 1.09$. This value still has a very large uncertainty, coming not only from the uncertainties on the radius, but also from the difficulties of measuring the frequency separation with observations from the ground.

The local estimation of this relation is also affected by systematic contributions due to deviations from the scaling law resulting from changes in the physics. Figure 9 shows that the change of the mixing length introduces a deviation from the scaling, giving an error of less than $0.01 M_{\odot}$ for the estimation of the stellar mass.

\section{Results}

We presented a detailed HRD analysis of $\beta H y d$ by taking the latest observational data for this stars. If we assume that the helium abundance of $\beta \mathrm{Hyd}$ should be higher than the primordial helium ( $Y \in[0.23,0.24]$; Peimbert et al. 2002 and references), and lower than the highest value of the helium determined for Population I stars with similar ages and masses as $\alpha$ Centauri A (Guenther \& Demarque 2000; Morel et al. 2000), we can infer that the helium abundance of $\beta \mathrm{Hyd}$ should be between 0.25 and 0.30 . By using the relation (3) we can estimate that, for $\beta$ Hyd,

$\frac{M_{\mathrm{HRD}}}{M_{\odot}}=1.10_{-0.07}^{+0.04}$,

where the error in $Z / X$ is also included. From the analysis of the models in Table 3, it is clear that the age of $\beta H y d$ should be

$6.4 \mathrm{Gyr} \leq t_{\mathrm{HRD}} \leq 7.1 \mathrm{Gyr}$. 
These results are in agreement with previous determinations of Dravins et al. (1998). We point out that the accuracy obtained in mass is particularly interesting for a single star. Helium and metals diffusion do not change considerably these results.

The use of the large frequency separation can constrain the mass of the star if the radius is known with high precision. The present bolometric and spectroscopic observations give the determination of the radius with an uncertainty of about $4 \%$. At present, the large frequency separation is not known to better than $4.0 \%$. Consequently, the uncertainty on the value of the mass is still large, with the preliminary result giving that

$$
\frac{M_{\Delta \bar{v}}}{M_{\odot}}=1.09 \pm 0.22
$$

This estimation is also affected by deviations from the assumed homology scaling law, due the changes in the physics. In the case of convection, when changing the value of $\alpha$ from 1.4 to 1.8 , we found that such an effect is below $0.01 M_{\odot}$. If the large frequency separation is determined with a precision below $1 \%$ (which is expected to be achieved by the space missions in Asteroseismology), the result is dominated by the error on the Luminosity, which results in an uncertainty for the mass of about $14 \%$. This is an independent estimate of the value determined with the HRD analysis, as discussed above, and therefore extremely valuable to raise some of the degeneracy we have found.

We note that the quoted error bars (Eqs. (7) and (9)) for the mass of $\beta \mathrm{Hyd}$ do not include the unknown uncertainties on the calculation of the models used to estimate the mass from the observations. This contribution is expected to be different for the determination from the HRD analysis or for the result found with the seismic study of the star. Therefore, the fact that both values are consistent is of great relevance. We will need more detailed seismic data in order to confirm that this consistency remains if the error bar in Eq. (9) is reduced.

The seismic information we can have on a star is not limited to the large frequency separation. The signature from the region of the second ionization of helium can be a valuable tool to constrain the value of $Y$. Such a test will require all individual frequencies (for $n$ between 8 and 24) with an accuracy of about $0.1 \mu \mathrm{Hz}$. With such data we can also expect to be able to provide an estimate of the mass/size (or equivalently - the age) for the helium core of the star.

\section{Conclusions}

In this work we have recalculated the best models for adjusting $\beta$ Hyd in the HR Diagram. In doing so, a detailed study of the uncertainty in the adjusting parameters has been done in order to evaluate the size of the parameter space that holds compatible solutions for the observed luminosity and effective temperature. The modelling parameters which have been evaluated are the helium abundance $(Y)$, the stellar mass $(M)$, the age $(t)$, the mixing length parameter $(\alpha)$ and the overshooting parameter $\left(\alpha_{\mathrm{ov}}\right)$. For the case of $\beta \mathrm{Hyd}$, an evolved solar-type star, we have found that the models are basically insensitive to convection parameters. The HRD analysis does not allow us to determine these parameters. Similar results were found for the helium abundance and stellar mass as a result of the strong degeneracy in $(Y, M)$.

Given the recent seismic observations of this star, and the planned missions in Asteroseismology, we have discussed the possibility of using seismic observations to test some of the modelling parameters. In particular we have aim at investigating the possibility of removing the $(Y, M)$ degeneracy. The use of the large frequency separation can provide that extra dimension to the HRD, which, at least partially, will remove some of the degeneracy by providing a direct determination of the mass. In this work we have in particular focused on how the mass and the age, and consequently the helium abundance, could be constrained by the observations. By taking into consideration the seismic observations already available for $\beta H y d$, and what we expect to have in the near future, we have shown that with accurate seismic data for $\beta H y d$ we may provide an extremely important post-main sequence calibration point on the evolution of a solar-type star.

The fact that $\beta H y d$ is an evolved star represents an extra difficulty in applying the traditional seismic tools for the analysis of the global parameters of the star. But the different behaviour of the oscillations for such a type of stars can also be used successfully to test the relevant aspects of their interiors, namely the age, or equivalently, the size of the central helium core as the star moves towards the red-giant phase.

Acknowledgements. This work was supported in part by Fundação para a Ciência e a Tecnologia - Portugal, through projects PESO/P/PRO15128/1999, ESO/FNU/43658/2001 and POCTI/1999/FIS/34549. JF gratefully thanks Pierre Morel for making the CESAM code available and to David Valls-Gabaud who instaled it at the Coimbra Observatory.

\section{References}

Alexander, D. R., \& Fergunson, J. W. 1993, ApJ, 437, 879

Aizenman, M., Smeyers, P., \& Weigert A. 1977, A\&A, 58, 41

Baglin, \& The COROT Team 1998, in New Eyes to See Inside the Sun and Stars, ed. F.-L. Deubner, J. Christensen-Dalsgaard, \& D. W. Kurtz (Kluwer), 301

Bahcall, J. N., \& Pinsonneault, M. H. 1995, Rev. Mod. Phys., 67, 781

Bedding, T. R., Butler, R. P., Kjeldsen, H., et al. 2001, ApJ, 549, L105

Blackwell, D. E., \& Lynas-Gray, A. E. 1998, A\&AS, 129, 505

Bohm-Vitense, E. 1958, Zs. Ap, 46, 108

Carrier, F., Bouchy, F., Kienzle, F., et al. 2001, A\&A, 378, 142

Castro, S., Porto de Mello, G. F., \& da Silva, L. 1999, MNRAS, 305, 693

Caughlan, G. R., \& Fowler, W. A. 1988, Atomic Data Nuc. Data Tables, 40, 284

Christensen-Dalsgaard, J. 1982, MNRAS, 199, 735

Christensen-Dalsgaard, J. 1991, in Challenges to theories of the structure of moderate-mass stars, ed. D. O. Gough, \& J. Toomre (Springer-Verlag), 11

Christensen-Dalsgaard, J., Bedding, T. R., \& Kjeldsen, H. 1995, ApJ, 443, L29

Cox, J. P. 1980, Theory of Stellar Pulsation (Princeton University Press, Princeton), 165

Cayrel de Strobel, G., Soubiran, C., \& Ralite, N. 2001, A\&A, 373, 159 
Castellani, V., Ciacio, F., Degl'Innocenty, S., et al. 1997, A\&A, 322, 801

Chmielewski, Y. 2000, A\&A, 353, 666

DiBenedetto 1998, A\&A, 339, 858

Dravins, D., Lindegren, L., \& VandenBerg, D. A. 1998, A\&A, 330, 1077

Dravins, D., Lindegren, L., Nordlund, A., \& Vandenberg, D. A. 1993, ApJ, 403, 385

Dravins, D., \& Nordlund, A. 1990, A\&A, 228, 203

Dziembowski, W. A., Fiorentini, G., Ricci, B., \& Sienkiewicz, R. 1999, A\&A, 343, 990

Eggleton, P. P., Faulkner, J., \& Flannery, B. P. 1973, A\&A, 23, 325

Favata, F., Micela, G., \& Sciortino, S. 1997, A\&A, 323, 809

Favata, F., Roxburgh, I. W., \& Galadí-Enríquez D., (eds) 2002, Stellar Structure and Habitable Planet Finding, ESA-SP 485 (ESA Publications Division, The Netherlands)

Fernandes, J., Lebreton, Y., Baglin, A., \& Morel, P. 1998, A\&A, 338, 455

Grevesse, N., \& Noels, A. 1993, in Origin and Evolution of the Elements, ed. N. Prantzos, E. Vangioni-Flam, \& M. Cassé (Cambridge University Press)

Guenther, D. B., \& Demarque, P. 2000, ApJ, 531, 503

Houdek, G., \& Rogl, J. 1996, BASI, 24, 317

Iglesias, C. A., \& Rogers, F. J. 1996, ApJ, 464, 943

Kjeldsen, H., Bedding, T. R., \& Christensen-Dalsgaard, J. 2000, in The Impact of Large-Scale Surveys on Pulsating Star Research, ed. L. Szabados, \& D. Kurtz, ASP Conf. Ser., 203, 73

Lebreton, Y., Auvergne, M., Morel, P., et al. 1993, In Inside the Stars, IAU Coll. 137, ed. A. Baglin, \& W. W. Weiss, ASP Conf. Ser., 40, 474
Lebreton, Y., Perrin, M.-N., Cayrel, R., Baglin, A., \& Fernandes, J. 1999, A\&A, 350, 587

Maeder, A., \& Meynet, G. 2000, ARA\&A, 38, 143

Matthews, J. M., et al. 2000, in The Impact of Large-Scale Surveys on Pulsating Star Research, ed. L. Szabados, \& D. Kurtz, ASP Conf. Ser., 203, 74

Monteiro, M. J. P. F. G., \& Thompson, M. J. 1998, in New Eyes to See Inside the Sun and Stars, ed. F.-L. Deubner, J. ChristensenDalsgaard, \& D. W. Kurtz (Kluwer), IAU Symp., 185, 317

Monteiro, M. J. P. F. G., Christensen-Dalsgaard, J., \& Thompson, M. J. 2002, ed. F. Favata, I. W. Roxburgh, \& D. Galadì-Enríquez, ESA SP-485 (ESA Publications Division, The Netherlands), 291

Morel, P. 1997, A\&AS, 124, 597

Morel, P., Provost, J., \& Berthomieu, G. 1997, A\&A, 327, 349

Morel, P., Provost, J., Lebreton, Y., et al. 2000, A\&A, 363, 675

Morel, P., Berthomieu, G., Provost, J., \& Thévenin, F. 2001, A\&A, 379,245

Montalban, J., \& Schatzman, E. 2000, A\&A, 354, 943

Noels, A., Grevesse, N., Magain, P., et al. 1991, A\&A, 247, 91

Peimbert, A., Peimbert, M., \& Luridiana, V. 2002, ApJ, 565, 668

Perryman, M. A. C., Lindegren, L., Kovalevsky, J., et al. 1997, A\&A, 323, 49

Randich, S., Gratton, R., Pallavicini, R., et al. 1999, A\&A, 348, 487

Ribas, I., Jordi, C., \& Giménez, A. 2000, MNRAS, 318, 55

Richer, J., Michaud, G., \& Turcotte, S. 2000, ApJ, 529, 338

Skuljan, J., Hearnshaw, J. B., \& Cottrell, P. L. 2000, PASP, 112, 966

van Altena, W. F., Lee, J. T., \& Hoffleit, E. D. 1995, The General Catalogue of Trigonometric Stellar Parallaxes, fourth edn., Yale Univ. Obs., Vol. I 